\title{
ERRATUM
}

\section{Evaluation of pastures for horses grazing on soils polluted by trace elements}

\section{P. Madejón · M. T. Domínguez $\cdot$ J. M. Murillo}

Published online: 10 March 2009

(C) Springer Science+Business Media, LLC 2009

\section{Erratum to: Ecotoxicology DOI 10.1007/s10646-009-0296-3}

The author regrets for error in Table 2 of his article. Correct version of Table 2 is as given below.

Table 2 Species composition of the pastures (affected and control sites)

\begin{tabular}{|c|c|c|c|c|c|c|c|}
\hline Family & Sp. & Spring & Autumn & Family & Sp. & Spring & Autumn \\
\hline \multirow[t]{12}{*}{ Poaceae } & Agrostis pourretii & 0.08 & 0.35 & Fabaceae & Biserrula pelecinus & 0.01 & \\
\hline & Avena barbata subsp. barbata & 0.03 & & & Medicago doliata & 0.06 & 0.01 \\
\hline & Avena sterilis & 0.1 & 0.05 & & Medicago murex & 0.07 & 0.01 \\
\hline & Bromus diandrus & 0.17 & 0.11 & & Medicago polymorpha & 0.13 & 0.03 \\
\hline & Bromus lanceolatus & 0.21 & & & Scorpiurus muricatus & 0.04 & 0.04 \\
\hline & Bromus madritensis & 0.1 & & & Vicia villosa subsp. varia & 0.02 & \\
\hline & Cynodon dactylon & 0.01 & 0.25 & Others & Anagallis arvensis & 0.07 & 0.01 \\
\hline & Hordeum marinum & 0.06 & & & Beta vulgaris & 0.01 & \\
\hline & Lamarckia aurea & 0.03 & & & Convulvulus arvensis & 0.01 & 0.06 \\
\hline & Lolium perenne & 0.1 & & & Echium plantagineum & & 0.05 \\
\hline & Phalaris minor & 0.05 & 0.03 & & Equisetum arvense & 0.01 & 0.03 \\
\hline & Piptatherum miliaceum & 0.08 & 0.1 & & Euphorbia helioscopia & 0.01 & \\
\hline \multirow[t]{12}{*}{ Asteraceae } & Andryala integrifolia & 0.1 & & & Heliotropum europaeum & & \\
\hline & Carduus pycnocephalus & 0.02 & & & Hirchsfeldia incana & & \\
\hline & Chrysanthemum coronarium & 0.09 & & & Malva hispanica & 0.04 & 0.05 \\
\hline & Coleostephus myconis & 0.21 & 0.12 & & Plantago coronopus & 0.01 & 0 \\
\hline & Conyza bonariensis & 0.05 & & & Plantago lagopus & 0.05 & 0 \\
\hline & Galactites tomentosa & 0.02 & & & Ranunculus trilobus & 0.03 & 0 \\
\hline & Lactuca serriola & 0.01 & & & Scirpoides holoschoenus & & 0.02 \\
\hline & Leontodon longirostris & 0.09 & 0.02 & & Torilis arvensis & 0.04 & 0.07 \\
\hline & Senecio lividus & 0.22 & 0.01 & & & & \\
\hline & Senecio vulgaris & 0.14 & 0.16 & & & & \\
\hline & Silybum marianum & 0.03 & 0.02 & & & & \\
\hline & Xanthium strumarium & & 0.01 & & & & \\
\hline
\end{tabular}

The relative frequency of observations of each species $(N=100)$ during the spring and autumn sampling is indicated

The online version of the original article can be found under doi:10.1007/s10646-009-0296-3.

P. Madejón $(\varangle) \cdot$ M. T. Domínguez · J. M. Murillo

Instituto de Recursos Naturales y Agrobiología de Sevilla (IRNAS), CSIC, P.O. Box 1052, 41080 Sevilla, Spain

e-mail: pmadejon@irnase.csic.es 